\title{
Hyers-Ulam Stability for Mackey-Glass and Lasota Differential Equations
}

\author{
Maher Nazmi Qarawani ${ }^{1}$ \\ ${ }^{1}$ Department of Mathematics, AlQuds Open University, Palestine \\ Correspondence: Maher Nazmi Qarawani, Department of Mathematics, AlQuds Open University, West-Bank, \\ Salfit P.O. Box 37, Palestine. E-mail: mkerawani@qou.edu
}

Received: September 12, 2012 Accepted: December 2, 2012 Online Published: January 8, 2013

doi:10.5539/jmr.v5n1p34 URL: http://dx.doi.org/10.5539/jmr.v5n1p34

\begin{abstract}
This paper considers the stability of differential equation of Mackey-Glass type in the sense of Hyers and Ulam with initial condition. It also considers the Hyers-Ulam stability of Lasota equation with initial condition. Some illustrative examples are given.
\end{abstract}

Keywords: Hyers-Ulam stability, Mackey-Glass, lasota, differential equation

\section{Introduction}

Ulam (1940) proposed the stability problem of functional equations during a talk before a Mathematical Colloquium at the University of Wisconsin, Madison. Hyers in his study (1941) solved the problem for approximately additive mappings, on Banach spaces. Rassias (1978) has generalized the result obtained by Hyers.

Alsina and Ger (1998) were the first mathematicians who investigated the Hyers-Ulam stability of the differential equation $g^{\prime}=g$. They proved that if a differentiable function $y: I \rightarrow R$ satisfies $\left|y^{\prime}-y\right| \leq \varepsilon$ for all $t \in I$, then there exists a differentiable function $g: I \rightarrow R$ satisfying $g^{\prime}(t)=g(t)$ for any $t \in I$ such that $|g-y| \leq 3 \varepsilon$, for all $t \in I$. This result of Alsina and Ger has been generalized by Takahasi et al. (2002) to the case of the complex Banach space valued differential equation $y^{\prime}=\lambda y$.

Furthermore, the results of Hyers-Ulam stability of differential equations of first order were also generlaized by Miura et al. (2003), Jung (2005) and Wang et al. (2008). Li and Shen (2009) proved the stability of nonhomogeneous linear differential equation of second order in the sense of the Hyers and Ulam $y^{\prime \prime}+p(x) y^{\prime}+q(x) y+r(x)=0$, while Gavruta et al. (2011) proved the Hyers-Ulam stability of the equation $y^{\prime \prime}+\beta(x) y=0$ with boundary and initial conditions. The author in his studies $(2012 \mathrm{a} \& \mathrm{~b})$ established the Hyers-Ulam stability of nonlinear differential equations of second order with initial conditions.

In this paper we investigate the Hyers-Ulam stability of Mackey-Glass nonlinear differential equation of first order:

$$
x^{\prime}+\gamma x=\frac{\beta x(t-\tau)}{1+x^{n}(t-\tau)}
$$

with the initial function $x(t)=\varphi(t), \forall t \leq 0$, and the initial condition

$$
x(0)=0
$$

where $\varphi(t)$ is positive and continuous $\forall t<0, x \in C^{1}(I), I=[a, b], 0<a<b<\infty, \beta, \gamma, n, \tau>0$. Moreover, we consider the Hyers-Ulam stability of Lasota nonlinear differential equation

$$
x^{\prime}+\gamma x=\beta e^{-x(t-\tau)} x^{n}(t-\tau), \quad \beta, \gamma, n, \tau>0
$$

with the initial function $x(t)=\varphi(t), \forall t \leq 0$, and the initial condition

$$
x(0)=0
$$

where $\varphi\left(t\right.$ is positive and continuous $\forall t<0, x \in C^{1}(I), I=[a, b], 0<a<b<\infty, \beta, \gamma, n, \tau>0$. 
Equation (1) was proposed by Mackey and Glass (1977) as a model of hematopoiesis (blood cell production), while Equation (3) was used by Lasota (1977).

It should be noted that in the present paper we apply a similar approach to that one used by the author in (2012a \& b).

Definition 1 We say that Equation (1) has the Hyers-Ulam stability with initial conditions (2) if there exists a positive constant $K>0$ with the following property:

For every $\varepsilon>0, x \in C^{1}[a, b]$, if

$$
\left|x^{\prime}+\gamma x-\frac{\beta x(t-\tau)}{1+x^{n}(t-\tau)}\right| \leq \varepsilon
$$

and $x(0)=0$, then there exists some $w \in C^{1}[a, b]$ satisfying the Equation (1) and $w(0)=0$, such that $|w(t)-x(t)| \leq$ $K \varepsilon$.

Definition 2 We say that Equation (3) has the Hyers-Ulam stability with initial conditions (4) if there exists a positive constant $K>0$ with the following property: For every $\varepsilon>0, x \in C^{1}(I)$, if

$$
\left|x^{\prime}+\gamma x-\beta e^{-x(t-\tau)} x^{n}(t-\tau)\right| \leq \varepsilon
$$

and $x(0)=0$, then there exists some solution $w \in C^{1}(I)$ of the Equation (3) and $w(0)=0$, such that

$$
|w(t)-x(t)| \leq K \varepsilon .
$$

\section{On Hyers-Ulam Stability of Equations}

Theorem 1 Suppose that $x: I \rightarrow \mathbb{R}$ is a twice continuously differentiable function. If $\beta b e^{\gamma \tau}<1$, then the Equation (1) has the Hyers-Ulam stability with initial condition (2).

Proof. Suppose that $\varepsilon>0$ and $x \in C^{1}(I)$ satisfies the inequality (5) and the initial condition $x(0)=0$. We will show that there exists a function $w(t) \in C^{1}(I)$ satisfying the Equation (1) such that $|x(t)-w(t)| \leq K \varepsilon$ and $w(0)=0$, where $K$ is a constant that never depends on $\varepsilon$ nor on $w(t)$. Substituting $x(t)=u(t) e^{-\gamma t}$ into the Equation (1), we get

$$
u^{\prime}(t) e^{-\gamma t}=\frac{\beta u(t-\tau) e^{-\gamma(t-\tau)}}{1+u^{n}(t-\tau) e^{-\gamma n(t-\tau)}}
$$

From the Definition 1 and the Equation (7) we obtain

$$
-\varepsilon \leq u^{\prime}(t) e^{-\gamma t}-\frac{\beta u(t-\tau) e^{-\gamma(t-\tau)}}{1+u^{n}(t-\tau) e^{-\gamma n(t-\tau)}} \leq \varepsilon
$$

Multiplying the inequality (8) by $e^{\gamma t}$, and then integrating with respect to $t$, we get

$$
-\frac{\varepsilon}{\gamma}\left(e^{\gamma t}-1\right) \leq u(t)-\int_{0}^{t} \frac{\beta u(s-\tau) e^{\gamma \tau}}{1+u^{n}(s-\tau) e^{-\gamma n(s-\tau)}} d s \leq \frac{\varepsilon}{\gamma}\left(e^{\gamma t}-1\right)
$$

Since $\varphi(t)>0$, then from (7) $u^{\prime}(t)>0, \forall t>0$, and hence the function $u(t)>0$, and is increasing $\forall t>0$. Now Let $M=\max _{a \leq t \leq b}\{u(t): t>0\}$. Thus, from (9) we have

$$
u(t)-\beta e^{\gamma \tau} \int_{0}^{t} u(s) d s<u(t)-\int_{0}^{t} \frac{\beta u(s-\tau) e^{\gamma \tau}}{1+u^{n}(s-\tau) e^{-\gamma n(s-\tau)}} d s \leq \frac{\varepsilon}{\gamma}\left(e^{\gamma t}-1\right)
$$

Hence

$$
M\left(1-\beta b e^{\gamma \tau}\right)<\frac{\varepsilon}{\gamma}\left(e^{\gamma t}-1\right)
$$

Therefore $M<K \varepsilon$, where $K=\frac{\left(e^{\gamma b}-1\right)}{\gamma\left(1-\beta b e^{\gamma \tau}\right)}, \beta b e^{\gamma \tau}<1$. So by virtue of the substitution $x(t)=u(t) e^{-\gamma t}$ we infer that

$$
\max _{a \leq t \leq b}(x(t)) \leq K \varepsilon
$$


Obviously, $w(t) \equiv 0$ is a solution of the Equation (1) satisfying the initial condition (2) and such that $|x(t)-w(t)| \leq$ $k \varepsilon$, which completes the proof.

Now we give an example illustrating the Theorem 1.

Example 1 Consider the equation

$$
x^{\prime}+0.001 x=\frac{0.009 x(t-9)}{1+x^{20}(t-9)}, 0 \leq t \leq 100
$$

with the initial condition

$$
x(0)=0
$$

and the history function $\varphi(t)=-t, t \in[-9,0]$.

Substituting $x(t)=u(t) e^{-t}$ into Equation (10), we get

$$
u^{\prime}(t) e^{-t}=\frac{0.009 u(t-9) e^{-(t-9)}}{1+u^{20}(t-9) e^{-(t-9)}}
$$

Multiplying the inequality

$$
-\varepsilon \leq u^{\prime}(t) e^{-t}-\frac{0.009 u(t-9) e^{-(t-9)}}{1+u^{20}(t-9) e^{-(t-9)}} \leq \varepsilon
$$

by $e^{t}$ and integrating from zero to $t$, we obtain

$$
u(t)-\frac{9 e^{0.009}}{10} \int_{0}^{t} u(s) d s<u(t)-\int_{0}^{t} \frac{0.009 u(s-9) e^{-(t-9)}}{1+u^{20}(s-9) e^{-(s-9)}} d s \leq \varepsilon\left(e^{t}-1\right)
$$

Hence

$$
M\left(1-\frac{9 e^{0.009}}{10}\right)<\varepsilon\left(e^{100}-1\right)
$$

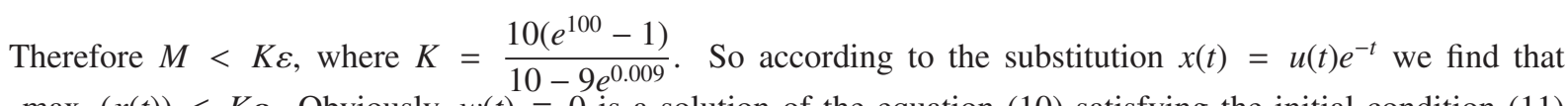
$\max _{0 \leq t \leq 100}(x(t)) \leq K \varepsilon$. Obviously, $w(t) \equiv 0$ is a solution of the equation (10) satisfying the initial condition (11) and such that $|x(t)-w(t)| \leq k \varepsilon$.

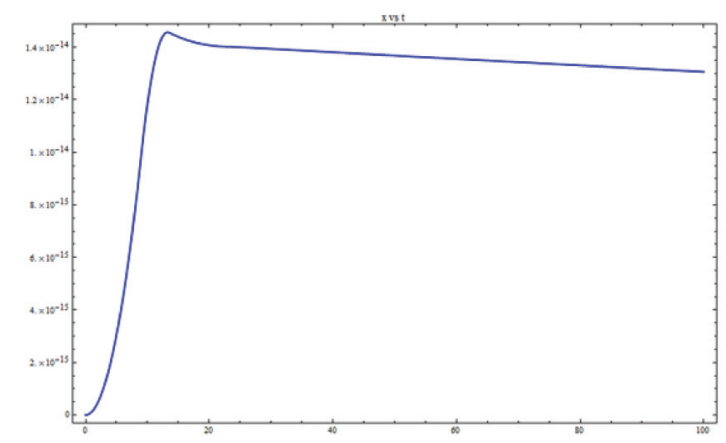

Figure 1. Solution of Equation (10) for $\gamma=0.001, \beta=0.009, \tau=9, n=20$

Hence, the problem (10-11) is stable in the sense of Hyers and Ulam (see Figure 1).

Remark 1 It should be noted that the condition $\beta b e^{\tau}<1$ in the Theorem 1 is sufficient; but it is not necessary for stability of the problem (1-2). The following example illustrates this fact.

Example 2 Consider the equation

$$
x^{\prime}+x=\frac{2 x(t-2)}{1+x(t-2)}, 0 \leq t \leq 250
$$

and the inequality

$$
\left|x^{\prime}+x-\frac{2 x(t-2)}{1+x(t-2)}\right| \leq \varepsilon
$$


with the initial condition $x(0)=0$, and the history function $\varphi(t)=-t, t \in[-2,0]$.

First, we notice that the condition $\beta b e^{\tau}<1$ in the Theorem 1 is not satisfied, because $500 e^{2}>1$, but the Equation (12) is still Hyers-Ulam stable (see Figure 2).

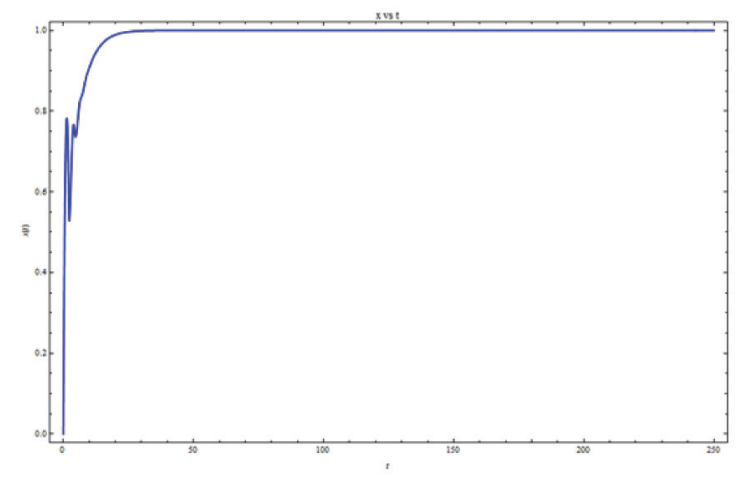

Figure 2. Solution of Equation (12) with $\gamma=1, \beta=2, \tau=2, n=1$

It is easy to check that for a given $\varepsilon>0$, the function $x(t)=\left(\frac{\varepsilon}{\varepsilon+1}\right)\left(1-e^{-t}\right)$ satisfies the inequality (13) and the zero initial condition $x(0)=0$. Indeed,

$$
\begin{aligned}
x^{\prime}+x-\frac{2 x(t-2)}{1+x(t-2)} & =\frac{\varepsilon e^{-t}}{\varepsilon+1}+\frac{\varepsilon}{\varepsilon+1}-\frac{\varepsilon e^{-t}}{\varepsilon+1}-\frac{2 \varepsilon\left(1-e^{-(t-2)}\right)}{\varepsilon+1+\varepsilon\left(1-e^{-(t-2)}\right)} \\
& =\frac{\varepsilon}{\varepsilon+1}-\frac{2 \varepsilon\left(1-e^{-(t-2)}\right)}{\varepsilon+1+\varepsilon\left(1-e^{-(t-2)}\right)} \\
& \leq \frac{\varepsilon}{\varepsilon+1}-\frac{2 \varepsilon\left(1-e^{-(t-2)}\right)}{2 \varepsilon+1}<\frac{\varepsilon}{\varepsilon+1}<\varepsilon
\end{aligned}
$$

On the other hand

$$
\begin{aligned}
x^{\prime}+x-\frac{2 x(t-2)}{1+x(t-2)}>x^{\prime}+x-2 x(t-2)>x^{\prime}-x \\
\\
=\frac{2 \varepsilon e^{-t}}{\varepsilon+1}-\frac{\varepsilon}{\varepsilon+1}>-\frac{\varepsilon}{\varepsilon+1}>-\varepsilon
\end{aligned}
$$

and $M=\operatorname{Sup}\left\{\left(\frac{\varepsilon}{\varepsilon+1}\right)\left(1-e^{-t}\right): t>0\right\}=\frac{\varepsilon}{\varepsilon+1}$.

Obviously, $w(t) \equiv 0$ is a solution of the Equation (12) satisfying the initial condition $w(0)=0$, and such that $|x(t)-w(t)| \leq K \varepsilon$, with $K=1$. Hence, the Equation (12) is stable in the sense of Hyers and Ulam.

Theorem 2 If $x: I \rightarrow \mathbb{R}$ is a twice continuously differentiable function and $\beta b e^{n \gamma \tau}[n] !<1$, then the Equation (3) has the Hyers-Ulam stability with initial conditions (4).

Proof. Suppose that $\varepsilon>0$ and $x \in C^{1}(I)$ satisfies the in Equation (6) and the initial conditions (4).

We will show that there exists a function $w(t) \in C^{1}(I)$ satisfying the Equation (3) such that $|x(t)-w(t)| \leq K \varepsilon$ and $w(0)=0$, where $K$ is a constant that never depends on $\varepsilon$ nor on $w(t)$.

Let

$$
x(t)=u(t) e^{-\gamma t}
$$

Setting (14) into Equation (3), we obtain

$$
u^{\prime}(t) e^{-\gamma t}=\beta u^{n}(t-\tau) e^{-u(t-\tau) e^{-\gamma(t-\tau)}} e^{-\gamma n(t-\tau)}
$$

Since $\varphi(t)>0$, then from $(15) u^{\prime}(t)>0, \forall t>0$, and hence the function $u(t)>0$, and is increasing $\forall t>0$.

From the inequality (6) and Equation (15) we obtain

$$
-\varepsilon \leq u^{\prime}(t) e^{-\gamma t}-\beta u^{n}(t-\tau) e^{-u(t-\tau) e^{-\gamma(t-\tau)}} e^{-\gamma n(t-\tau)} \leq \varepsilon
$$


Multiplying the inequality (16) by $e^{\gamma t}$, and then integrating with respect to $t$, we get

$$
-\frac{\varepsilon}{\gamma}\left(e^{\gamma t}-1\right) \leq u(t)-\int_{0}^{t} \beta u^{n}(t-\tau) e^{-u(t-\tau) e^{-\gamma(t-\tau)}} e^{-\gamma n(t-\tau)} e^{\gamma t} d s \leq \frac{\varepsilon}{\gamma}\left(e^{\gamma t}-1\right)
$$

It follows that

$$
u(t)-\beta e^{n \gamma \tau} \int_{0}^{t} \frac{u^{n}(s)}{e^{u(s)}} d s<u(t)-\int_{0}^{t} \beta u^{n}(t-\tau) e^{-u(t-\tau) e^{-\gamma(t-\tau)}} e^{-\gamma n(t-\tau)} e^{\gamma t} d s \leq \frac{\varepsilon}{\gamma}\left(e^{\gamma t}-1\right)
$$

Let $M=\max _{a \leq t \leq b}\{u(t): t>0\}$.

For $M$ we have two cases:

I) $M \leq 1$;

II) $M>1$.

If $M \leq 1$ then

$$
\frac{u^{n}(s)}{e^{u(s) e^{\gamma \tau}}} \leq u \leq M \leq[n] ! M
$$

and clearly, we get that

$$
M \leq \frac{\varepsilon\left(e^{\gamma b}-1\right)}{\gamma\left(1-\beta b e^{n \gamma \tau}[n] !\right)}
$$

where $\beta b e^{n \gamma \tau}[n] !<1$.

Assume that inf $\left\{1, \frac{\varepsilon\left(e^{\gamma b}-1\right)}{\gamma\left(1-\beta b e^{n \gamma \tau}[n] !\right)}\right\}=m$, then $\max _{a \leq t \leq b}(x(t)) \leq m \leq \frac{\varepsilon\left(e^{\gamma b}-1\right)}{\gamma\left(1-\beta b e^{n \gamma \tau}[n] !\right)}$.

Now let $M>1$. Then we obtain

$$
\frac{u^{n}(s)}{e^{u(s) e^{\gamma \tau}}}=\frac{u^{n}}{1+u e^{\gamma \tau}+\frac{u e^{2 \gamma \tau}}{2 !}+\ldots+\frac{u^{k} e^{k \gamma \tau}}{k !}+\ldots} \leq \frac{[n] ! u^{[n]+1}}{u^{[n]} e^{[n] \gamma \tau}} \leq[n] ! M
$$

Hence, setting the last inequality into (15) we get

$$
M\left(1-\beta b e^{n \gamma \tau}[n] !\right)<\frac{\varepsilon}{\gamma}\left(e^{\gamma t}-1\right)
$$

Therefore $M<K \varepsilon$, where $K=\frac{\left(e^{\gamma b}-1\right)}{\gamma\left(1-\beta b e^{n \gamma \tau}[n] !\right)}$, where $[n]$ is the greatest integer function, and $\beta b e^{n \gamma \tau}[n] !<1$. So by virtue of the substitution $x(t)=u(t) e^{-\gamma t}$ we infer that

$$
\max _{a \leq t \leq b}(x(t)) \leq K \varepsilon
$$

Clearly $w(x) \equiv 0$, is a trivial solution of Equation (3) that satisfies the initial condition $w(0)=0$ and $|x(t)-w(t)| \leq$ $K \varepsilon$, which completes the proof.

Example 3 Consider Lasota equation for the parameters $\beta=0.004, \gamma=1, \tau=1, n=\ln 10$

$$
x^{\prime}+x=0.004 e^{-x(t-1)}[x(t-1)]^{\ln 10}, \quad t \in[0,10]
$$

with the initial function $\varphi(t)=0.5, t \in[-1,0]$ and the zero initial condition

$$
x(0)=0
$$

Applying the same arguments used in the proof of Theorem 2 for this case, we obtain that $\max _{a \leq t \leq b}(x(t)) \leq K \varepsilon$, where $K=\frac{\left(e^{10}-1\right)}{\left(1-0.004 *[\ln 10] ! * 10 e^{\ln 10}\right)}=5\left(e^{10}-1\right)$ such that $0.004 * 20 e^{\ln 10}=\frac{1}{2}<1$. It is clear that $w(x) \equiv 0$, is 
a trivial solution of the Equation (18) with the initial condition $w(0)=0$, and $|x(t)-w(t)| \leq K \varepsilon$. Therefore, the problem (18-19) is stable in the sense of Hyers and Ulam.

Remark 2 It should be noted that the condition $\beta b e^{n \tau}[n] !<1$ in the Theorem 2 is sufficient; but it is not necessary for stability of the problem (3-4).

To prove this assumption, consider $x^{\prime}+x=e^{-x(t-1)} x(t-1)$, with $x(0)=0$. Applying the same argument used in Example 2, we can show that the function $x(t)=\frac{\varepsilon}{2}\left(1-e^{-t}\right)$ satisfies the inequality

$$
\left|x^{\prime}+x-e^{-x(t-1)} x(t-1)\right| \leq \varepsilon
$$

and $M=\operatorname{Sup}\left\{\frac{\varepsilon}{2}\left(1-e^{-t}\right): t>0\right\}=\frac{\varepsilon}{2}<\varepsilon$. Obviously, $w(t) \equiv 0$ is a solution of the given equation satisfying the initial condition $x(0)=0$ and such that $|x(t)-w(t)| \leq \varepsilon$, the proof is complete.

\section{Nicholson's Blowflies Equation}

Consider Nicholson's Blowflies Equation which is a special case (when $n=1$ ) of Lasota differential equation

$$
x^{\prime}+\gamma x=\beta e^{-x(t-\tau)} x(t-\tau), \quad \beta, \gamma, \tau>0
$$

with the initial function $x(t)=\varphi(t)$, where $\varphi(t)>0, \forall t<0$, and the initial condition

$$
x(0)=0
$$

Theorem 1 Suppose that $x: I \rightarrow \mathbb{R}$ is a twice continuously differentiable function. If $\beta b e^{\gamma \tau}<1$, then the Equation (20) has the Hyers-Ulam stability with the initial conditions (21).

Proof. Applying the same arguments used in the proof of Theorem 2 to the case when $n=1$, we obtain

$$
u(t)-\beta e^{\gamma \tau} \int_{0}^{t} \frac{u(s)}{e^{u(s)}} d s<u(t)-\int_{0}^{t} \beta u(t-\tau) e^{-u(t-\tau) e^{-\gamma(t-\tau)}} e^{-\gamma(t-\tau)} e^{\gamma t} d s \leq \frac{\varepsilon}{\gamma}\left(e^{\gamma t}-1\right)
$$

Hence

$$
M \leq \frac{\varepsilon\left(e^{\gamma b}-1\right)}{\gamma\left(1-\beta b e^{\gamma \tau}\right)}
$$

Since $\beta b e^{n \gamma \tau}<1$ then by virtue of the substitution $x(t)=u(t) e^{-\gamma t}$ we infer that

$$
\max _{a \leq t \leq b}(x(t)) \leq K \varepsilon
$$

It is obvious that $w(x) \equiv 0$, is a trivial solution of Equation (20) that satisfies (21) and $|x(t)-w(t)| \leq K \varepsilon$. Hence, the problem (20-21) is stable in the sense Hyers and Ulam.

\section{Conclusion}

Here we have established the Hyers-Ulam stability of Mackey-Glass and Lasota delay differential equations with initial conditions. The results are achieved by integrating the differential equations and then estimating the maximum of solutions. The plots produced by Mathematica have verified our theoretical results.

\section{Acknowledgements}

The author is deeply indebted to anonymous referees and editors for careful and thoughtful reading of the manuscript and useful suggestions which helped to improve the presentation of results.

\section{References}

Alsina, C., \& Ger, R. (1998). On some inequalities and stability results related to the exponential function. J. Inequal. Appl., 2, 373-380.

Gavruta, P., Jung, S. \& Li, Y. (2011). Hyers-Ulam Stability For Second-Order Linear Differential Equations With Boundary Conditions. Electronic Journal of Differential Equations, 2011(80), 1-7. Retrieved from http://ejde.math.txstate.edu/Volumes/2011/80/gavruta.pdf

Hyers, D. H. (1941). On the stability of the linear functional equation. Proceedings of the National Academy of Sciences of the United States of America, 27, 222-224. http://dx.doi.org/10.1073/pnas.27.4.222 
Jung, S. M. (2005). Hyers-Ulam stability of linear differential equations of first order. J. Math. Anal. Appl., 311, 139-146. http://dx.doi.org/10.1016/j.jmaa.2005.02.025

Lasota, A. (1977). Ergodic problems in biology. Dynamical systems, II, pp. 239-250, Warsaw. Astérisque, No. 50. Soc. Math. France, Paris.

Li, Y., \& Shen, Y. (2009). Hyers-Ulam Stability of Nonhomogeneous Linear Differential Equations of Second Order. International Journal of Mathematics and Mathematical Sciences, 2009, Article ID 576852, 7. http://dx.doi.org/ doi:10.1155/2009/576852

Mackey, M. C., \& Glass, L. (1977). Oscillation and chaos in physiological control systems. Science, 197(4300), 287-289.

Miura, T., Miyajima, S., \& Takahasi, S.-E.(2003). A characterization of Hyers-Ulam stability of first order linear differential operators. J. Math. Anal. Appl., 286, 136-146. http://dx.doi.org/10.1016/S0022-247X(03)00458$\mathrm{X}$

Qarawani, M. N. (2012a). Hyers-Ulam Stability of Linear and Nonlinear Differential Equations of Second Order. International Journal of Applied Mathematics, 1(4), 422-432.

Qarawani, M. N. (2012b). Hyers-Ulam Stability of a Generalized Nonlinear Second- Order Differential Equation. Applied Mathematics, 3(12), USA (to be published on December).

Rassias, T. M. (1978). On the stability of the linear mapping in Banach spaces. Proceedings of the American Mathemaical Society, 72(2), 297-300. http://dx.doi.org/10.1090/S0002-9939-1978-0507327-1

Takahasi, E., Miura, T., \& Miyajima, S. (2002). On the Hyers-Ulam stability of the Banach space-valued differential equation $y^{\prime}=\lambda y$. Bulletin of the Korean Mathematical Society, 39(2), 309-315. http://dx.doi.org/10.4134/BKMS.2002.39.2.309

Ulam, S. M. (1964). Problems in Modern Mathematics. New York, NY, USA: John Wiley \& Sons.

Wang, G., Zhou, M., \& Sun, L. (2008). Hyers-Ulam stability of linear differential equations of first order. Appl. Math. Lett., 21, 1024-1028. 